\title{
西 \\ Gastric electrical stimulation relieves nausea and vomiting in the long term
}

Long-term relief from intractable nausea and vomiting can be achieved with gastric electrical stimulation (GES) according to a small study published in Digestive and Liver Disease. "Gastric electrical stimulation was effective over 5 years in patients who would have otherwise been treated with a gastrectomy," notes author Guillaume Gourcerol from the University of Rouen, France.

Chronic nausea and/or vomiting can be clinically challenging to treat, especially when symptoms are refractory to standard treatment with antiemetic and prokinetic agents. In the past decade, GES has been investigated as an alternative treatment for these types of intractable nausea and vomiting. "This therapy was efficient in the short term," says Gourcerol, "but information on the long-term effects was lacking, especially in European centers."

In this study, 31 patients with chronic nausea and vomiting (either normal or delayed gastric emptying) who were refractory to standard treatment were fitted with a device that delivers highfrequency electrical pulses in the gastric greater curvature. Individuals were then assessed using symptomatic and quality of

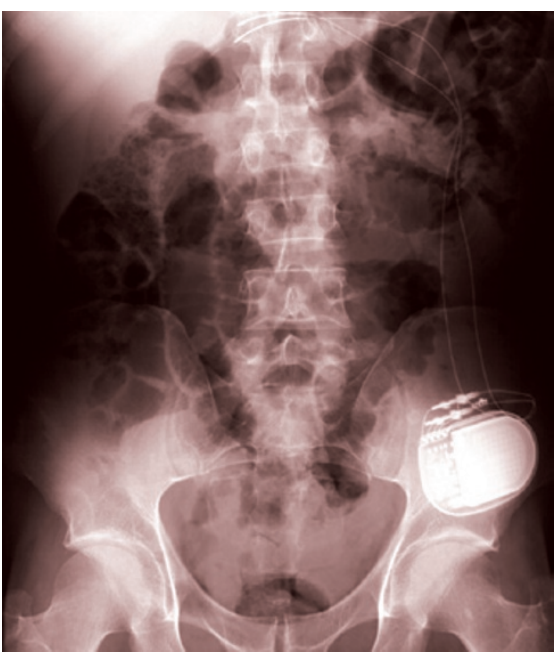

life scores at baseline, and 6 months and 5 years after implantation.

Of the 20 patients assessed after 5 years, dyspeptic symptoms-vomiting, nausea, satiety, bloating and epigastric pain-were markedly improved after the start of GES. Improvements were also observed in patient's quality of life scores (by $27 \%$, $P<0.01)$. In addition, GES was well tolerated, with only one infection associated with the device reported in the study period and only mild to moderate transient discomfort or pain at the implantation site.

The authors acknowledge that further studies are warranted and have now initiated a randomized double-blind clinical trial $(n=220)$ to confirm their findings.

\section{Katrina Ray}

Original article Gourcerol, G. et al. Long term efficacy of gastric electrical stimulation in intractable nausea and vomiting. Dig. Liver Dis. doi:10.1016/j.dld.2012.01.013 